\title{
Tyrosine aminotransferase: biochemical and structural properties and molecular dynamics simulations
}

\author{
Prajwalini Mehere ${ }^{1}$, Qian Han ${ }^{1}$, Justin A. Lemkul ${ }^{1}$, Christopher J. Vavricka ${ }^{1,2}$, Howard Robinson ${ }^{3}$, \\ David R. Bevan ${ }^{1}$, Jianyong $\mathrm{Li}^{1}{ }^{1}$ \\ ${ }^{1}$ Department of Biochemistry, Virginia Tech, Blacksburg, VA 24061, USA \\ 2 CAS Key Laboratory of Pathogenic Microbiology and Immunology, Institute of Microbiology, Chinese Academy of Sciences, \\ Beijing 100101, China \\ ${ }^{3}$ Biology Department, Brookhaven National Laboratory, Upton, NY 11973, USA \\ $\bowtie$ Correspondence: lij@vt.edu \\ Received October 11, 2010 Accepted October 19, 2010
}

\section{ABSTRACT}

Tyrosine aminotransferase (TAT) catalyzes the transamination of tyrosine and other aromatic amino acids. The enzyme is thought to play a role in tyrosinemia type II, hepatitis and hepatic carcinoma recovery. The objective of this study is to investigate its biochemical and structural characteristics and substrate specificity in order to provide insight regarding its involvement in these diseases. Mouse TAT (mTAT) was cloned from a mouse cDNA library, and its recombinant protein was produced using Escherichia coli cells and purified using various chromatographic techniques. The recombinant mTAT is able to catalyze the transamination of tyrosine using $\alpha$-ketoglutaric acid as an amino group acceptor at neutral $\mathrm{pH}$. The enzyme also can use glutamate and phenylalanine as amino group donors and p-hydroxyphenylpyruvate, phenylpyruvate and alpha-ketocaproic acid as amino group acceptors. Through macromolecular crystallography we have determined the MTAT crystal structure at $2.9 \AA$ resolution. The crystal structure revealed the interaction between the pyridoxal-5'-phosphate cofactor and the enzyme, as well as the formation of a disulphide bond. The detection of disulphide bond provides some rational explanation regarding previously observed TAT inactivation under oxidative conditions and reactivation of the inactive TAT in the presence of a reducing agent. Molecular dynamics simulations using the crystal structures of Trypanosoma cruzi TAT and human TAT provided further insight regarding the substrate-enzyme interactions and substrate specificity. The biochemical and structural properties of TAT and the binding of its cofactor and the substrate may help in elucidation of the mechanism of TAT inhibition and activation.

KEYWORDS tyrosine aminotransferase, crystal structure, substrate specificity, tyrosine, tyrosinemia

\section{INTRODUCTION}

Tyrosine aminotransferase (TAT) catalyzes reversible transamination of tyrosine and $p$-hydroxyphenylpyruvate (pHPP) (al-Hemidan and al-Hazzaa, 1995). TAT deficiency leads to tyrosinemia type II in humans due to genetic mutation (Natt et al., 1987). In tyrosinemia type II, tyrosine accumulates in blood, eventually causing lesions in the patients (al-Hemidan and al-Hazzaa, 1995). In humans, more than 15 mutations have been identified in the patients of tyrosinemia type II in Italy, France, USA and the UK (Natt et al., 1992; Endo, 1998; Charfeddine et al., 2006; Maydan et al., 2006; Minami-Hori et al., 2006; Meissner et al., 2008; Pasternack et al., 2009). Tyrosinemia type II is often associated with consanguinity. The incidence is less than one in 250,000 (Macsai et al., 2001). Eye, skin, and neurological symptoms are the foremost features of the disease (Pasternack et al., 2009). The disease is associated with microcephaly, tremor, ataxia, self-mutilating behavior, fine motor coordination disturbances, language deficits, and convulsions (Bein and Goldsmith, 1977; Cavelier-Balloy et al., 1985).

Trypanosoma cruzi TAT (tcTAT) shares over $40 \%$ sequence identity with the mammalian TATs. T. cruzi causes 
Chagas disease, a parasitic disease that is endemic in Latin America. It has been shown that tcTAT may be involved in drug resistance to benznidazole, a drug currently used in chemotherapy of Chagas disease (Rego et al., 2008).

TAT is also considered as a marker of mature hepatocytes (Touboul et al., 2010). Recently, Fu and colleagues demonstrated that TAT is a novel tumor suppressor gene and its inactivation, caused by gene deletion and hypermethylation, contributes to the pathogenesis of hepatocellular carcinoma (Fu et al., 2010).

Previous studies showed that TAT is a homodimer and is composed of two identical polypeptide chains (Blankenfeldt et al., 1999). It is expressed not only in the liver but also in the kidneys (Hargrove and Mackin, 1984) and brain (Hargrove and Mackin, 1984). However the enzyme has the highest activity in the liver. In hTAT, the first 38 amino acids may not be involved in enzyme dimerization, and are not required in the active site stability and enzyme-substrate interactions (Sobrado et al., 2003). This N-terminal fragment, however, is required for being targeted by the ubiquitin-proteosome pathway (Gross-Mesilaty et al., 1997), which degrades proteins to small peptides (Ciechanover et al., 2000). So far crystal structures of TATs from Escherichia coli (Ko et al., 1999), T. cruzi (Blankenfeldt et al., 1999), and Homo sapiens (Protein Data Bank code, 3dyd) have been reported. In this study, we provide a mouse TAT (mTAT) crystal structure and its biochemical characteristics for comparative study. All crystal structures of TATs lack substrate binding information; therefore we used molecular dynamics simulations to provide further insight into substrate-enzyme interactions and substrate specificity.

\section{RESULTS AND DISCUSSION}

\section{Biophysical and biochemical characteristics}

Using affinity, Source-Q and gel-filtration chromatography, we purified the mTAT recombinant protein to a high purity as confirmed by SDS-PAGE. The purified MTAT showed high TAT activity using a-ketoglutaric acid (KGA) as an amino

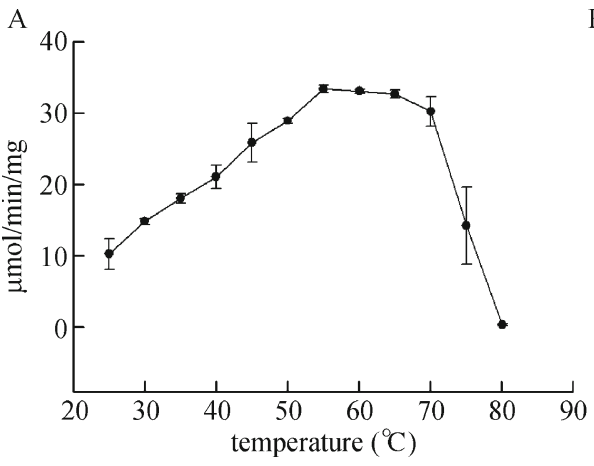

group acceptor. Among the temperature points tested, mTAT showed the highest TAT activity at $50-70^{\circ} \mathrm{C}$ (Fig. 1A). When testing for optimum $\mathrm{pH}$ across a range of $\mathrm{pH}$ 6.0-11.0, mTAT displayed the highest activity at pH 7.0 (Fig. 1B). Sixteen $\alpha$ keto acids (listed in MATERIALS AND METHODS section) were tested for their potential as amino group acceptors for mTAT with $5 \mathrm{mM}$ tyrosine ( $5 \mathrm{mM}$ glutamate for pHPP) as the amino group donor. The enzyme only showed detectable activity toward KGA, pHPP, phenylpyruvate and $\alpha$ ketocaproic acid. The enzyme kinetic study determined the enzyme kinetic parameters toward these four keto-acids (Table 1). MTAT does not have detectable activity toward glycine, alanine, and aspartate. Since hTAT showed transamination activity toward glutamate, phenylalanine as well as tyrosine (Sivaraman and Kirsch, 2006), kinetic parameters of mTAT toward these three amino acids were also tested and calculated (Table 1). Based on the mTAT substrate specificity, mTAT behaves in a manner similar to hTAT, in that it has a narrow substrate specificity compared to tcTAT (Nowicki et al., 2001; Sobrado et al., 2003; Sivaraman and Kirsch, 2006; Donini et al., 2009). TcTAT, on the other hand, has broad substrate specificity; in particular, it has an affinity to alanine, which differs considerably from tyrosine, the primary substrate of mTAT and hTAT (Nowicki et al., 2001).

\section{Overall structure}

The crystal structure of mTAT was determined by molecular replacement using hTAT structure (PDB code, 3dyd) as a model and refined to $2.9 \AA$ resolution. The final model contains 378 amino acid residues and yields a crystallographic $\mathrm{R}$ value of $26.3 \%$ and an $\mathrm{R}$-free value of $29.5 \%$ (Table 2). The first 23 residues at the $\mathrm{N}$-terminal end are disordered, and missed in the structure. All residues in the structure are in allowed regions of the Ramachandran plot as defined with PROCHECK (Laskowski et al., 1993). The structure has a large domain and a small domain. The large domain (residues 86-334) contains an eight-stranded beta sheet, and the small domain comprises the C-terminal part (residues 335-442) and a small fragment in the $\mathrm{N}$-terminal

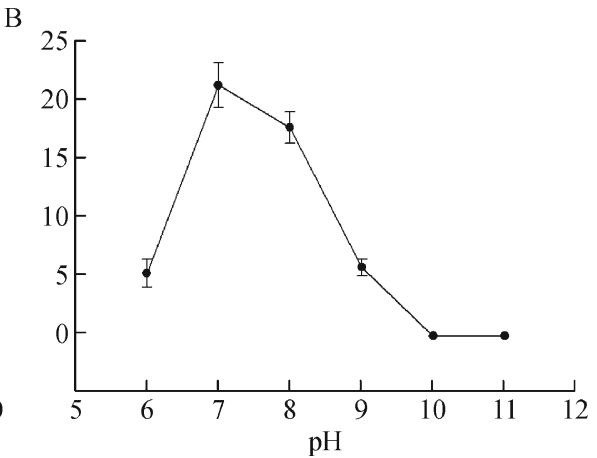

Figure 1. Effect of temperature and pH on enzyme activity. The activities of recombinant $\mathrm{mTAT}(\mathrm{A})$ at different temperatures and (B) at different $\mathrm{pH}$ values. 
Table 1 Kinetic parameters of mTAT toward a-keto acids

\begin{tabular}{|c|c|c|c|}
\hline & $\begin{array}{c}K_{m} \\
(\mathrm{mM})\end{array}$ & $\begin{array}{c}k_{\text {cat }} \\
\left(\min ^{-1}\right)\end{array}$ & $\begin{array}{c}k_{\text {cat }} / K_{m} \\
\left(\min ^{-1} \mathrm{mM}^{-1}\right)\end{array}$ \\
\hline \multicolumn{4}{|c|}{ amino acid substrates } \\
\hline tyrosine & $1.8 \pm 0.5$ & $1160 \pm 240$ & 640 \\
\hline glutamate & $4.9 \pm 0.8$ & $560 \pm 40$ & 120 \\
\hline phenylalanine & $11.4 \pm 4.1$ & $920 \pm 210$ & 80 \\
\hline \multicolumn{4}{|l|}{ keto acid substrates } \\
\hline pHPP & $0.7 \pm 0.4$ & $950 \pm 260$ & 1360 \\
\hline KGA & $1.8 \pm 0.2$ & $1540 \pm 80$ & 850 \\
\hline phenylpyruvate & $0.9 \pm 0.1$ & $160 \pm 10$ & 180 \\
\hline$\alpha$-ketocaproic acid & $7.3 \pm 3.1$ & $110 \pm 30$ & 20 \\
\hline
\end{tabular}

The activities were measured as described in the MATERIALS AND METHODS section. The $K_{\mathrm{m}}$ and $k_{\text {cat }}$ for $\alpha$-keto acids were derived by using varying concentrations $(0.2-10 \mathrm{mM})$ of individual $\alpha$-keto acids in the presence of $5 \mathrm{mM}$ of tyrosine (10 mM glutamate for pHPP). The $K_{\mathrm{m}}$ and $k_{\text {cat }}$ for amino acids were derived by using varying concentrations $(0.2-5 \mathrm{mM})$ of individual amino acids in the presence of $10 \mathrm{mM}$ of KGA (or $2 \mathrm{mM}$ pHPP for glutamate). The parameters were calculated by fitting the Michaelis-Menten equation to the experimental data using the enzyme kinetics module. Results are means \pm SE.

Table 2 Data collection and refinement statistics of mTAT crystals

\begin{tabular}{|c|c|}
\hline crystal data & \\
\hline space group & $\mathrm{C} 222_{1}$ \\
\hline \multicolumn{2}{|l|}{ unit cell } \\
\hline$\alpha(\AA)$ & 69.3 \\
\hline$\beta(\AA)$ & 84.8 \\
\hline$Y(\AA)$ & 158.0 \\
\hline$\alpha=\beta=\gamma\left({ }^{\circ}\right)$ & 90 \\
\hline \multicolumn{2}{|l|}{ data collection } \\
\hline X-ray source & $\mathrm{BNL}^{\mathrm{a}}-\mathrm{X} 29$ \\
\hline wavelength $(\AA)$ & 1.0809 \\
\hline resolution $(\AA)^{\mathrm{b}}$ & $2.9(3.0-2.9)$ \\
\hline total number of reflections & 55966 \\
\hline number of unique reflections & 10641 \\
\hline R-merge ${ }^{b}$ & $8.6(38.8)$ \\
\hline $1 / s^{b}$ & $24.4(2.3)$ \\
\hline redundancy ${ }^{\mathrm{b}}$ & $6.0(4.1)$ \\
\hline completeness $(\%)^{\mathrm{b}}$ & $87.7(43.6)$ \\
\hline \multicolumn{2}{|l|}{ refinement statistics } \\
\hline R-work (\%) & 26.3 \\
\hline R-free (\%) & 29.5 \\
\hline RMS bond lengths $(\AA)$ & 0.017 \\
\hline RMS bond angles $\left({ }^{\circ}\right)$ & 1.718 \\
\hline No. of ligand or cofactor molecules ${ }^{c}$ & $1 \mathrm{LLP}$ \\
\hline No. of water molecules & 38 \\
\hline average B overall $\left(\AA^{2}\right)$ & 107.8 \\
\hline \multicolumn{2}{|l|}{ statistics on Ramachandran plot (\%) } \\
\hline most favored regions & 83.5 \\
\hline
\end{tabular}

(Continued)

\begin{tabular}{lc}
\hline crystal data & \\
\hline additional allowed regions & 15.9 \\
generously allowed regions & 0.6 \\
disallowed regions & 0 \\
\hline a Brookhaven National Laboratory. \\
${ }^{\mathrm{b}}$ The values in parentheses are for the highest resolution shell. \\
${ }^{\mathrm{c}}$ LLP, lysine-pyridoxal-5'-phosphate.
\end{tabular}

part (residues 64-85), which folds into a 2-stranded beta sheet covered with helices. The residue Asp247 interacts with the pyridine nitrogen of the cofactor (Fig. 2), which is structurally and functionally conserved within fold-type I of the pyridoxal-5-phosphate (PLP)-dependent enzyme family, indicating its importance for catalysis. Based upon the above features of the structure, mTAT is a fold-type I PLP-dependent enzyme (Mehta et al., 1993; Grishin et al., 1995; Jansonius, 1998; Käck et al., 1999; Schneider et al., 2000). A DALIbased search (Holm and Sander, 1993) revealed significant structural homology of mTAT with tcTAT (PDB code, 1bw0; RMSD, 1.2 A; number of aligned positions, 413) (Blankenfeldt et al., 1999) in the Protein Data Bank. All these TATs, together with most of kynurenine aminotransferases I and III, belong to the aminotransferase subfamily Iy (Rossi et al., 2004; Han et al., 2009; Han et al., 2010).

\section{Active site of mTAT}

Residual electron density clearly revealed the presence of covalently bound PLP in the active center (Fig. S2 and Fig. 2). The C4A atom of PLP is covalently attached to the NZ atom of Lys280 through the formation of an internal Schiff base, and the internal aldimine gives rise to residue LLP280, represented as sticks in Fig. 2. The PLP pyridoxal ring is stacked 


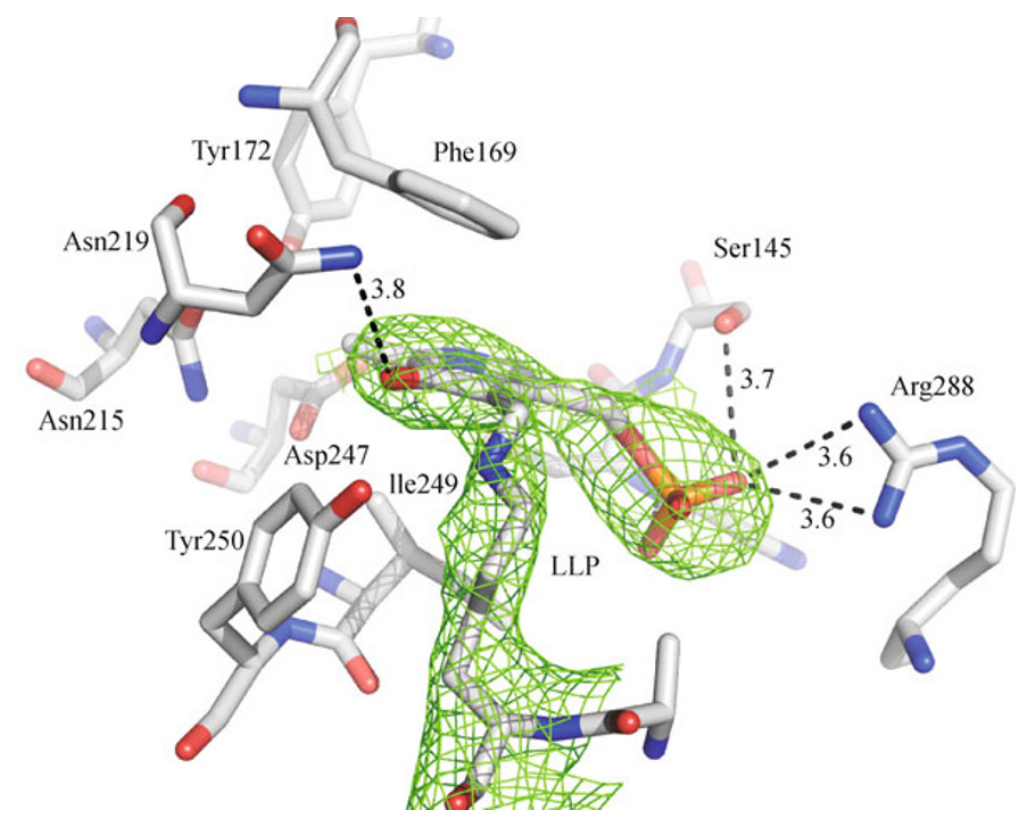

Figure 2. Active center. LLP (PLP bonded with Lys280) and residues within $4 \AA$ distance from PLP are shown in sticks. The 2 FoFC electron density map covering LLP is shown contoured at the 1.1 sigma level.

between residues 11 e249 and Phe169 by hydrophobic interactions, and the C2A atom of PLP exhibits a hydrophobic interaction with the Asn215 side chain. The side chain of Asn219 is hydrogen bonded to the $\mathrm{O} 3$ of the pyridoxal. The phosphate moiety of PLP is anchored by polar interactions with the peptide amide group of residue Cys144 as well as with the side chains of Ser145 and Arg288. A superposition of hTAT (PDB code 3dyd) and mTAT shows that the two proteins have the same architecture and that all residues around the PLP molecule (within $6 \AA$ ) are identical in the two enzymes. This suggests that hTAT and mTAT share the same PLP binding sites.

\section{Disulphide bond formation}

One disulphide bond is observed in the mTAT structure between Cys144 and Cys275 residues (Fig. S3 and Fig. 3A). Via superposition of hTAT and mTAT (RMSD, 0.6 ̊) we identified the residues within a $6 \AA$ range of PLP in both structures, and those residues having significant conformational difference are shown in Fig. 3B. Cys144 is shown to be in the active site, forming hydrogen bonds with the $\mathrm{O} 3, \mathrm{O} 1$ and N1 atoms of PLP. The hydrogen bonding interaction with the $\mathrm{N} 1$ atom of the PLP pyridine ring may interfere with the interaction between the PLP N1 atom and Asp247, which is
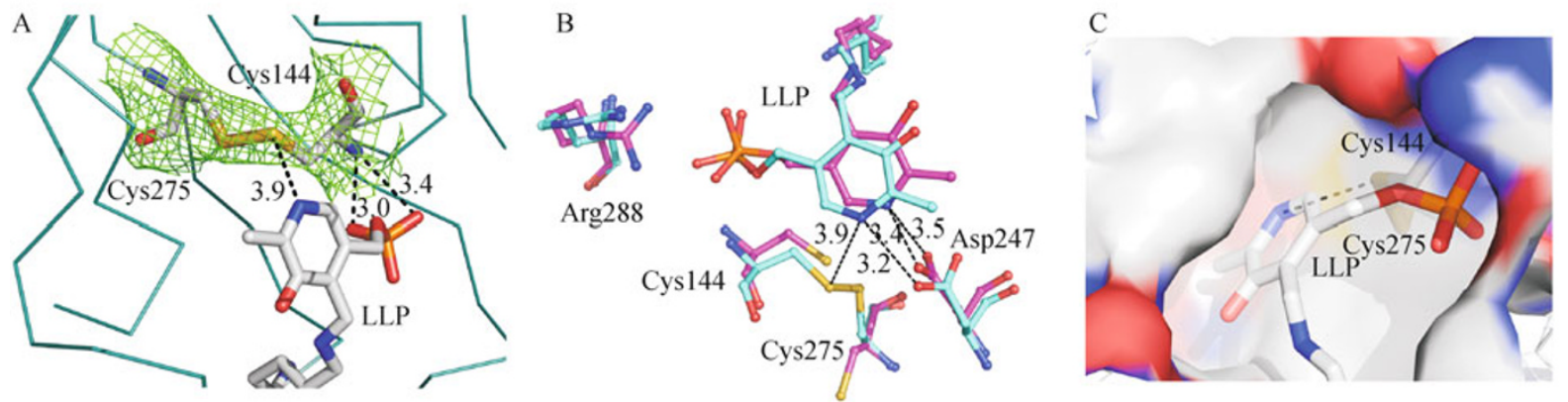

Figure 3. Disulphide bond formation. (A) LLP and the disulphide bond formed between Cys 144 and Cys 275 residues are shown in sticks. The $2 F \mathrm{~F}-F \mathrm{c}$ electron density map covering residues Cys 144 and Cys 275 is shown contoured at the 1.1 sigma level. (B) The mTAT structure is superimposed onto the hTAT structure. Residues having significant conformational difference within $6 \AA$ of PLP in the mTAT structure compared with the hTAT structure are shown. mTAT is colored in teal, and hTAT is in pink. (C) The enzyme active center cleft surface is colored by element (C: light gray, N: blue, O: red, S: yellow, P: orange); LLP and side chains of Cys 144 and Cys 275 are shown in sticks. 
critical to the transamination reaction. Therefore, the formation of this disulphide bond could be involved in the regulation of enzyme catalysis. The formation of an intra or inter TAT disulphide bond was long ago proposed as being involved in the inactivation of the enzyme (Beneking et al., 1978; Buckley and Milligan, 1978; Federici et al., 1978). An important feature of the observed inactivation was its reversibility by thiol compounds (Beneking et al., 1978; Federici et al., 1978). Our findings of a disulphide bond formation between Cys 144 and Cys275 residues support this previous suggestion. The thiol groups of Cys144 and Cys275 are on an enzyme active center cleft surface (Fig. 3C); therefore they are ready to be targeted by an oxidant or a reducing agent. Forming a disulphide bond can lead to a conformational change in Cys144, causing it to interact with the N1 atom of the PLP ring, which is probably the mechanism of TAT inactivation. Reducing agents may break the disulphide bond and change the Cys144 conformation back to an active form, which is the most likely explanation for the reversibility feature of the TAT inactivation. Site-directed mutagenesis of these two residues in future studies will shed light on their roles in protein inactivation.

\section{Substrate specificity of TATs revealed by molecular docking and dynamics simulation}

Molecular docking and molecular dynamics (MD) simulations were conducted in an attempt to identify structural features that contribute to substrate specificity. Free energy and clusters used of the docked complexes are shown in Table S1. Following MD simulations, principal components analysis was conducted on each of the systems in this study to explore the fundamental, lowfrequency vibrations of the protein structure that contribute to catalysis. No differences were observed in the vibrational modes of uncomplexed and complexed TAT enzymes, indicating that binding of tyrosine and alanine did not affect the structural stability of either hTAT or tcTAT. Fig. 4 illustrates the structures of the complexes in this study after $5 \mathrm{~ns}$ of MD simulation. In each case, a representative TAT structure was chosen to illustrate the protein, and the final positions of the ligands in each of the three simulations are superimposed onto this structure following rotational and translational fitting of the system to the reference structure. In the case of the hTAT-Ala complex (Fig. 4A), it is clear that the position of the ligand (alanine) is more variable than in the case of the tcTAT-Ala complex (Fig. 4C). The RMSD of the alanine ligand in hTAT is $0.75 \pm$ $0.16 \mathrm{~nm}$, averaged across all three replicate simulations, while in the case of the tcTAT-Ala complex, the RMSD of alanine is only $0.28 \pm 0.05 \mathrm{~nm}$. The RMSD indicates the level of deviation from the original docked pose, and another meaningful analysis is the root-mean-square fluctuation (RMSF) of the ligand, the magnitude of fluctuation about a mean position. Again, in the case of the hTAT-Ala complex, the bound alanine was more variable, with an average RMSF of $0.27 \pm 0.14 \mathrm{~nm}$, while that of the tcTAT-Ala ligand was $0.11 \pm 0.03 \mathrm{~nm}$. From these results, it can be seen that the bound alanine in the hTAT-Ala complex fluctuates within the active site substantially over the course of the MD simulations, such that it is not consistently positioned to interact with LLP in a way that is amenable to catalysis, while in the case of tcTAT the bound pose is more stable and positioned for catalysis.

The position of the bound tyrosine in the hTAT-Tyr complex deviated substantially from the initial docked pose (Fig. 4B), while that of tcTAT-Tyr remained more consistent (Fig. 4D). The RMSD of tyrosine in the hTAT-Tyr complex was $0.53 \pm$ $0.09 \mathrm{~nm}$, averaged over all three simulations, but the RMSF was very low, $0.11 \pm 0.03 \mathrm{~nm}$. These results indicate that docking tyrosine to hTAT resulted in the tyrosine ligand being placed in a local, rather than global, energy minimum, leading to large movements of tyrosine (as indicated by the RMSD), but that the final positions achieved in the MD simulations were stable (as indicated by the RMSF). In the tcTAT-Tyr complex, the bound tyrosine was moved very little, with an average RMSD of $0.25 \pm 0.09 \mathrm{~nm}$ and an average RMSF of $0.09 \pm 0.04$.

In both the hTAT-Tyr and tcTAT-Tyr complexes, the $\alpha$-amino group remained proximal to the imine functional group of the LLP cofactor, oriented in such a way that catalysis could be carried out. In the case of hTAT-Tyr, the average distance between the LLP imine and $\alpha$-amino group on tyrosine was $0.73 \pm 0.01 \mathrm{~nm}$, while that of tcTAT-Tyr was $0.64 \pm 0.10 \mathrm{~nm}$. In the hTAT-Ala complex, the distance between the LLP imine and the $\alpha$-amino group of alanine was, on average, $0.75 \pm$ $0.24 \mathrm{~nm}$, nearing $1.0 \mathrm{~nm}$ in one simulation. For tcTAT-Ala, this average distance was $0.68 \pm 0.04 \mathrm{~nm}$.

Careful inspection of nearby residues in the TAT active site reveals the reason for the more consistent binding pose of alanine in tcTAT relative to hTAT. In tcTAT, two hydrophobic residues, lle19 and Val40, provide a hydrophobic platform with which the $\beta$-carbon of alanine (as well as tyrosine) can interact favorably (Fig. 5). These residues are not present in the hTAT enzyme, allowing the alanine ligand greater mobility in the active site. This mobility is very likely the reason that hTAT cannot carry out catalysis on alanine as it does not bind stably in the proper orientation. In contrast, the binding of tyrosine to both tcTAT and hTAT is very stable. The position and orientation of tyrosine in the active site of both hTAT and tcTAT is largely unchanged in each simulation (Fig. 4). In the case of tcTAT, tyrosine forms hydrogen bonds with Arg20 while simultaneously interacting with lle19 and Val40 through hydrophobic interactions.

In the hTAT-Tyr complex, the bound tyrosine ligand is principally stabilized by interactions with Arg417 (both cation$\pi$ and hydrogen bonding) and Phe359 (aromatic stacking), as shown in Fig. 6. These interactions were observed in all three 

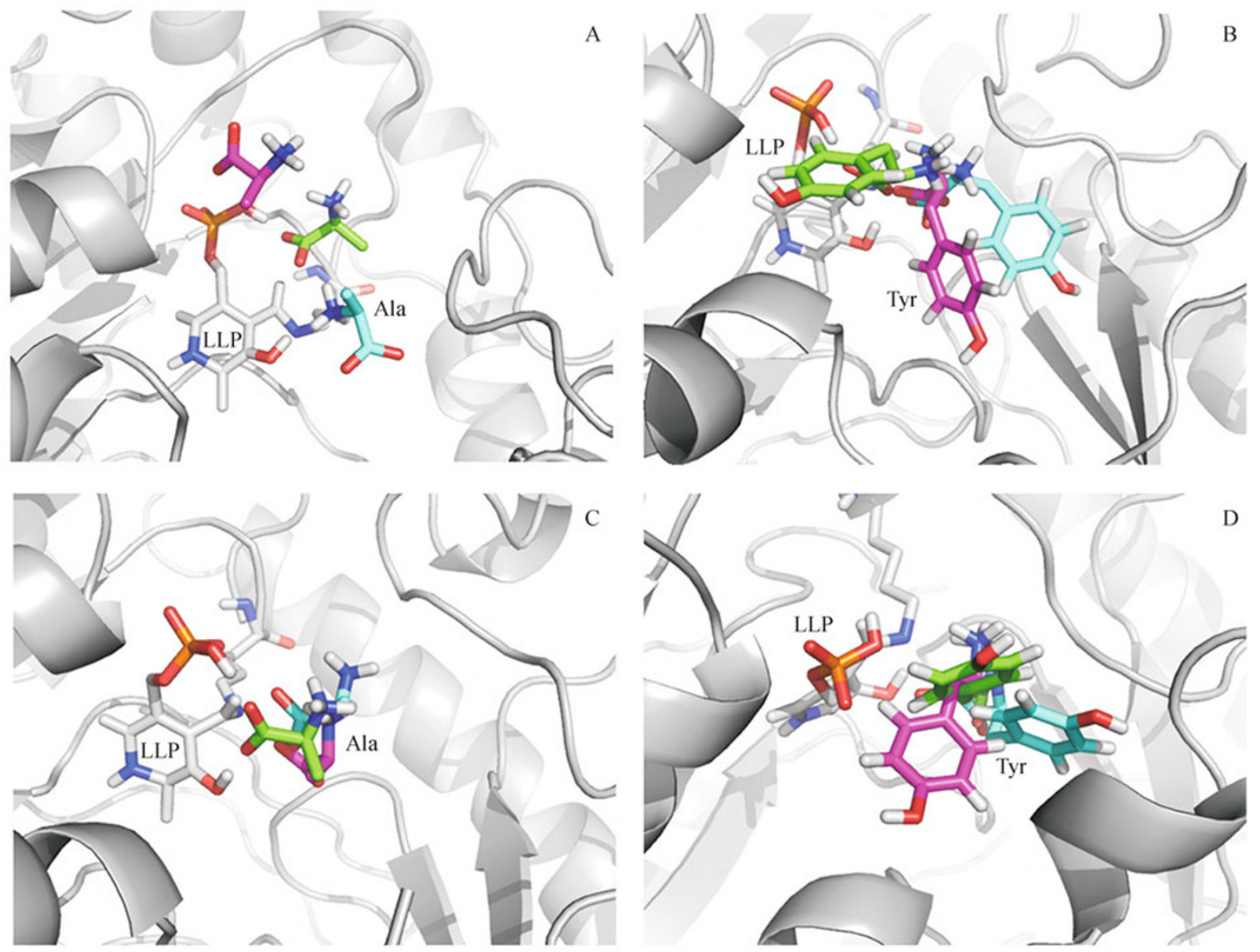

Figure 4. Structures of substrate bound TAT. Structures of (A) hTAT-Ala, (B) hTAT-Tyr, (C) tcTAT-Ala, and (D) tcTAT-Tyr complexes at the end of $5 \mathrm{~ns}$ of MD simulation. The TAT protein in each case is shown as a gray cartoon, with bound ligands shown as sticks and colored by element, with carbon atoms tinted green, cyan, and magenta to indicate the results of the different simulations. PLP and the covalently attached lysine are shown as sticks and colored by element, with gray-tinted carbon atoms.

simulations, but persisted in only two of the simulations. In the third simulation of the hTAT-Tyr complex, the tyrosine ligand oriented itself toward Phe169, a residue that is more distant from the active site. Although stable over much of the simulation, this interaction is likely an artifact of an incorrect initial docked pose. Tyrosine, by virtue of its size, is able to interact with Arg417 and Phe410, which are somewhat distant from the PLP cofactor. The distance of Phe410 from the active site prevents smaller ligands, like alanine, from forming stabilizing hydrophobic interactions while simultaneously remaining in position near the PLP cofactor.

In summary, the interaction of alanine and tyrosine ligands with tcTAT is stabilized by hydrophobic interactions involving lle19 and Val40. In tcTAT-Tyr, the tyrosine ligand is further stabilized by hydrogen bonding with Arg20. In the case of hTAT-Tyr, the tyrosine ligand forms favorable contacts with Phe410 and Arg417 at the periphery of the active site. Binding of alanine to hTAT, however, is not stable; as the smaller ligand cannot form stabilizing contacts with the more distant phenylalanines, and, unlike tyrosine, alanine cannot interact favorably with the guanidinium group of arginine.

\section{CONCLUSIONS}

We report herein the biochemical properties and crystal structure of mTAT. The crystal structure of mTAT demonstrated a disulphide bond formation, which is close enough to interact with $\mathrm{N} 1$ atom of the PLP pyridine ring, and may inhibit the enzyme reaction. This feature could well support a previously proposed mechanism of TAT inactivation mediated by disulphide bond formation. MD simulations predict that there are hydrophobic interactions in tcTAT that can be utilized by both alanine and tyrosine in binding to the enzyme, and that tyrosine is further stabilized by hydrogen bonding to a nearby arginine residue. In the case of hTAT, probably in mTAT as well, additional hydrophobic and hydrogen bonding interactions also promote the binding of tyrosine, but are too distant from the active site to be utilized by alanine. 


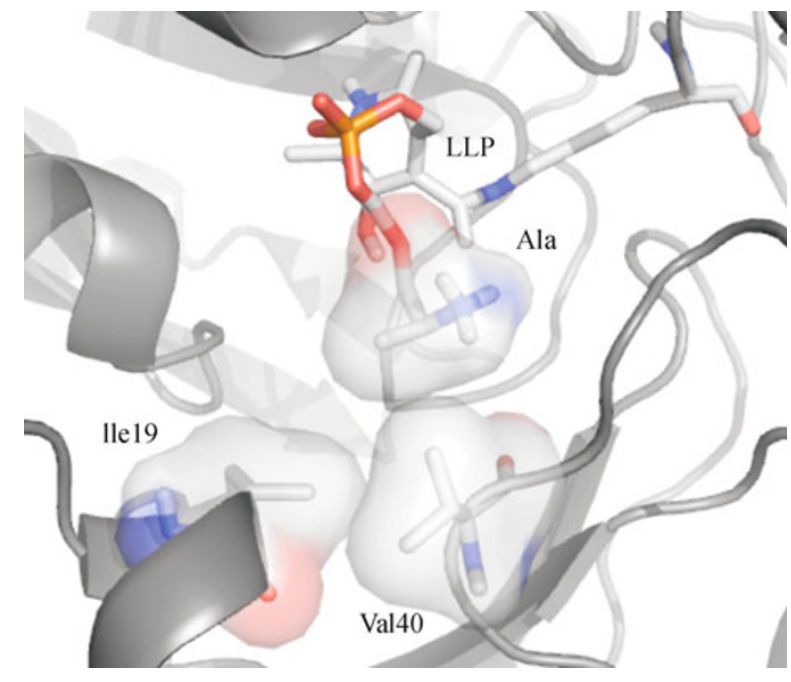

Figure 5. Detail of the tcTAT-Ala complex. The alanine ligand, lle19, and Val40 are shown as sticks with overlaid surfaces, and are colored by element. The pyridoxal 5'phosphate and covalently attached lysine are shown as sticks and colored by element.

\section{MATERIALS AND METHODS}

\section{Expression and purification of mTAT}

mTAT cDNA sequence was amplified from mouse liver cDNA pool using a forward primer (5'-CATATGAAGGCCAGGTGGAATGT-3') and reverse primer (5'-CTCGAGCTAGTAGTGCTGTTCACAGAACTC-3') containing Nde1 and Xho 1 restriction sites, respectively. The amplified sequence, encoding mTAT amino acid residues 41-442, was cloned into an Impact ${ }^{\mathrm{TM}}-\mathrm{CN}$ plasmid (New England Biolabs) for expression of a fusion protein containing a chitin binding domain. Transformed E. coli cells were cultured at $37^{\circ} \mathrm{C}$. After induction with $0.2 \mathrm{mM}$ isopropyl-1-thio- $\beta$-D-galactopyranoside, the cells were cultured at $15^{\circ} \mathrm{C}$ for $24 \mathrm{~h}$. Four liters of cells were harvested as the starting materials for affinity purification. The soluble fusion proteins were applied to a column packed with chitin beads and subsequently hydrolyzed under reducing conditions. The affinity purification resulted in the isolation of mTAT at around $80 \%$ purity. The recombinant mTAT was further purified by Source $Q$ ion-exchange and gel-filtration chromatography. The purified recombinant mTAT was concentrated to $10 \mathrm{mg} / \mathrm{mL}$ protein in $10 \mathrm{mM}$ phosphate buffer $(\mathrm{pH} 7.5)$ containing $40 \mathrm{mM}$ PLP using a Centricon YM-50 concentrator (Millipore). The concentrated protein was directly used in crystallization screening and optimization. Protein concentration was tested by a protein assay kit from Bio-Rad (Hercules, CA) using bovine serum albumin as a standard. $10 \%$ glycerol and $10 \mathrm{mM} \beta$ mercaptoethanol were added into the protein solution for the enzyme used in the biochemical characterization.

\section{Activity assay and enzyme kinetic study}

To determine the substrate specificity for $\alpha$-keto acids, $16 \alpha$-keto acids were individually tested for their ability to function as an amino group acceptor for mTAT using tyrosine as an amino group donor. Those include glyoxylate, indo-3-pyruvate, KGA, a-keto-methylthiobutyric acid, $\alpha$-ketoadipate, $\alpha$-ketobutyrate, $\alpha$-ketocaproic acid, $\alpha$-ketoisoleucine, $\alpha$-ketoleucine, $\alpha$-ketovalerate, $\alpha$-ketovaline, mercaptopyruvate, oxaloacetate, phenylpyruvate, pHPP, and pyruvate. Each of the $16 \alpha-$ keto acids were assayed at $2 \mathrm{mM}$ in the presence of $5 \mathrm{mM}$ tyrosine and $40 \mu \mathrm{M}$ PLP, prepared in $100 \mathrm{mM}$ phosphate buffer at $\mathrm{pH}$. The rate of pHPP production was determined using reversed phase highperformance liquid chromatography with ultraviolet detection (HPLCUV) at $295 \mathrm{~nm}$. To determine the optimum $\mathrm{pH}$ for mTAT-mediated transamination reactions, a phosphate and borate buffer mixture was adjusted to $\mathrm{pH}$ 6-11 used to prepare mTAT/Tyrosine/KGA reaction mixtures.

For enzyme kinetics studies, the transamination of tyrosine, phenylalanine, glutamate catalyzed by mTAT with phenylpyruvate, pHPP, KGA or a-ketocaproic acid as an amino group acceptor was tested using an assay mixture containing various concentrations of substrates, $40 \mathrm{mM}$ PLP, $100 \mathrm{mM}$ phosphate buffer $(\mathrm{pH} 7)$, and $1 \mu \mathrm{g}$ enzyme in a total volume of $100 \mu \mathrm{L}$. The mixture was incubated for $15 \mathrm{~min}$ at $38^{\circ} \mathrm{C}$ and stopped by adding an equal amount of absolute ethanol. The product was quantitated using HPLC with electrochemical detection (HPLC-ED) to measure the o-phthaldialdehyde thiol (OPT)-amino acid product conjugates after their corresponding reaction mixtures were derivatized by OPT reagent (Han et al., 2001).

\section{Crystallization, data collection and processing, and structure determination}

The crystals were grown by the hanging-drop vapor diffusion method with the volume of reservoir solution at $500 \mu \mathrm{L}$ and the drop volume at $2 \mu \mathrm{L}$, containing $1 \mu \mathrm{L}$ of protein sample and $1 \mu \mathrm{L}$ of reservoir solution. The optimized crystallization buffer consisted of $20 \%$ PEG 4000, 100 mM cacodylic acid buffer, $\mathrm{pH} 6.8$, and $20 \%$ glycerol. Diffraction data of mTAT crystal was collected at the Brookhaven National Synchrotron Light Source beam line X29A $(\lambda=1.0908 \AA)$. Data were collected using an ADSC CCD detector. All data were indexed and integrated using HKL-2000 software; scaling and merging of diffraction data were performed using the program SCALEPACK (Otwinowski and Minor, 1997). The parameters of the crystals and data collection are listed in Table 2. The structure of mTAT was determined by the molecular replacement method using hTAT structure (Protein Data Bank code, 3dyd). The program Molrep (Vagin and Teplyakov, 1997) was employed to calculate both the cross-rotation and translation functions of the model. The initial model was subjected to iterative cycles of crystallographic refinement with the Refmac 5.2 (Murshudov et al., 1997) and graphic sessions for model building using Coot (Krissinel and Henrick, 2004). The cofactor and substrate molecules were modeled before adding solvent molecules based on both the

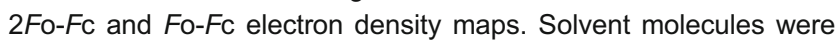
manually added and refined with Refmac 5.2. Superposition of structures was done using "SSM Superposition" function in Coot (Krissinel and Henrick, 2004). Figures were generated using Pymol (DeLano, 2002). Protein and substrate interaction was also analyzed using Pymol (DeLano, 2002).

\section{Docking and molecular dynamics simulations}

Complexes of both hTAT and tcTAT with alanine and tyrosine bound were obtained by molecular docking using AutoDock (Morris et al., 


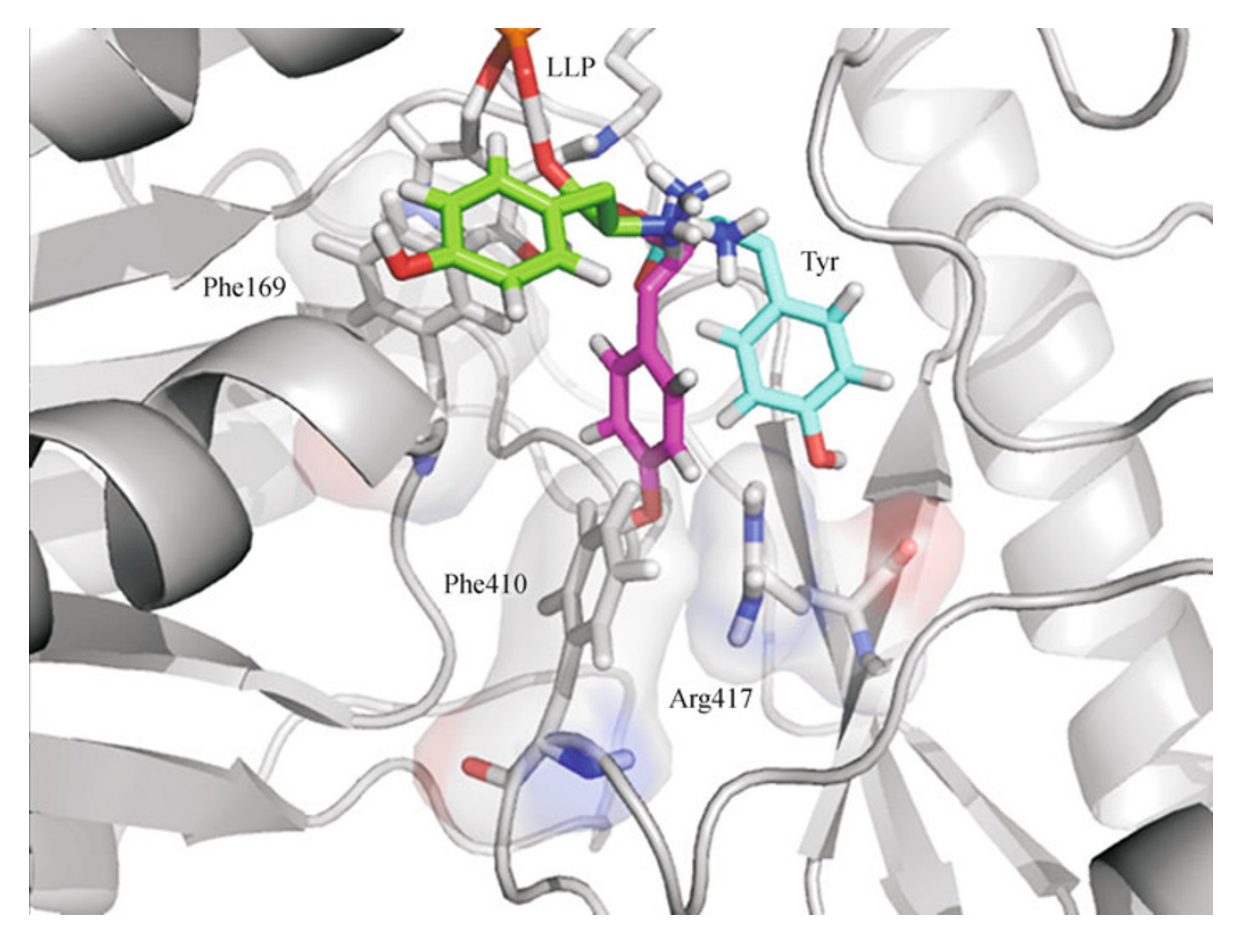

Figure 6. Interactions between tyrosine and the hTAT active site. Residues Phe169, Phe410, and Arg417 are shown as sticks and colored by element, with van der Waals surfaces overlaid to illustrate packing. The TATenzyme and bound tyrosine residues are depicted as in Fig. 4.

1998; Huey et al., 2007). The molecules for docking were prepared by using USCF Chimera (Pettersen et al., 2004) to add hydrogen atoms to the receptor and both ligands (alanine and tyrosine). AutoDock Tools 1.5.2 (ADT) was used to prepare the ligands and receptor, after which docking was performed using AutoDock 4.0 (Morris et al., 1998; Huey et al., 2007). The X, Y, and Z dimensions of the grid were set to $45 \times 45 \times 51 \AA$ with default grid spacing of $0.375 \AA$ around the cofactor of both proteins. The Lamarckian Genetic Algorithm (LGA) was used to predict binding positions, orientations, and conformations of the ligands. Default parameters were used, except for the number of energy evaluations, which was set to 250,000 . A total of 2000 docking poses (500 for each ligand docked to each protein) were generated and clustered with a $2.0 \AA$ threshold. The lowest energy configuration in the first cluster was considered the best docking pose for each ligand. These docked complexes were used as input for molecular dynamics simulations.

From each of these starting structures, three simulations were conducted. Systems were prepared and simulations conducted with GROMACS, version 4.0.5 (Hess et al., 2008). All structures were centered in a rhombic dodecahedral simulation cell filled with simple point charge (SPC) water (Berendsen et al., 1981) and sodium counterions to give an electrically net neutral system. The force field used was GROMOS96 53A6 (Oostenbrink et al., 2004). Parameters for the covalently bound pyridoxal 5'-phosphate cofactor were assigned based on analogous functional groups already present in the force field library. Following steepest descents minimization, the systems were equilibrated in two phases, during which position restraints were applied to all protein and cofactor heavy atoms. First, 100 picoseconds (ps) of constant volume (NVT) equilibration were performed at $310 \mathrm{~K}$, with temperature maintained by the Berendsen weak coupling method (Berendsen et al., 1984). Next, 100 ps of constant pressure (NPT) equilibration were performed, with temperature controlled by the Nosé-Hoover thermostat (Nose, 1984; Hoover, 1985) and pressure controlled by the Parrinello-Rahman barostat (Parrinello and Rahman, 1981; Nose and Klein, 1983). Following this phase, position restraints were removed and production simulations conducted for $5 \mathrm{~ns}$. During data collection, the same thermostat and barostat were used as in NPT equilibration. For all simulations, shortrange non-bonded interactions were truncated at $1.2 \mathrm{~nm}$ for computational efficiency. Dispersion correction was applied to energy and pressure terms to account for truncation of van der Waals terms. Long-range electrostatics were calculated with the Particle Mesh Ewald method (Darden et al., 1993; Essmann et al., 1995), using a cubic-spline interpolated grid, with 0.12-nm grid spacing. Constraints were applied to all bonds using the LINCS algorithm (Hess et al., 1997), allowing an integration time step of $2 \mathrm{fs}$.

\section{ACKNOWLEDGEMENTS}

This work was supported in part by a research grant from NINDS (NS062836) and carried out in part at the National Synchrotron Light Source, Brookhaven National Laboratory. We are grateful to Stephanie N. Lewis for helping with molecular docking.

\section{ABBREVIATIONS}

hTAT, human tyrosine aminotransferase; KGA, a-ketoglutaric acid; LLP, lysine-pyridoxal-5'-phosphate; mTAT, mouse tyrosine 
aminotransferase; pHPP, p-hydroxyphenylpyruvate; PLP, pyridoxal5'-phosphate; RMSD, root-mean-square deviation; TAT, tyrosine aminotransferase; tcTAT, Trypanosoma cruzi tyrosine aminotransferase

\section{REFERENCES}

al-Hemidan, A.I., and al-Hazzaa, S.A. (1995). Richner-Hanhart syndrome (tyrosinemia type II). Case report and literature review. Ophthalmic Genet 16, 21-26.

Bein, N.N., and Goldsmith, H.S. (1977). Recurrent massive haemorrhage from benign hepatic tumours secondary to oral contraceptives. Br J Surg 64, 433-435.

Beneking, M., Schmidt, H., and Weiss, G. (1978). Subcellular distribution of a factor inactivating tyrosine aminotransferase. Study of its mechanism and relationship to different forms of the enzyme. Eur J Biochem 82, 235-243.

Berendsen, H.J.C., Postma, J.P.M., Van Gunsteren, W.F., DiNola, A., and Haak, J.R. (1984). Molecular dynamics with coupling to an external bath. J Chem Phys 81, 3684-3690.

Berendsen, H.J.C., Postma, J.P.M., Van Gunsteren, W.F., and Hermans, J. (1981). Interaction models for water in relation to protein hydration. Jerusalem Symposia on Quantum Chemistry and Biochemistry 14, 331-342.

Blankenfeldt, W., Nowicki, C., Montemartini-Kalisz, M., Kalisz, H.M., and Hecht, H.J. (1999). Crystal structure of Trypanosoma cruzi tyrosine aminotransferase: substrate specificity is influenced by cofactor binding mode. Protein Sci 8, 2406-2417.

Buckley, W.T., and Milligan, L.P. (1978). Participation of cysteine and cystine in inactivation of tyrosine aminotransferase in rat liver homogenates. Biochem J 176, 449-454.

Cavelier-Balloy, B., Venencie, P.Y., Lemonnier, V., Verola, O., Servant, J.M., Puissant, A., and Civatte, J. (1985). Histiocytoid hemangioma of the scalp. Ann Dermatol Venereol 112, 965-972.

Charfeddine, C., Monastiri, K., Mokni, M., Laadjimi, A., Kaabachi, N., Perin, O., Nilges, M., Kassar, S., Keirallah, M., Guediche, M.N., et al. (2006). Clinical and mutational investigations of tyrosinemia type II in Northern Tunisia: identification and structural characterization of two novel TAT mutations. Mol Genet Metab 88, 184-191.

Ciechanover, A., Orian, A., and Schwartz, A.L. (2000). Ubiquitinmediated proteolysis: biological regulation via destruction. Bioessays 22, 442-451.

Darden, T., York, D., and Pedersen, L. (1993). Particle mesh Ewald: an N.log(N) method for Ewald sums in large systems. J Chem Phys 98, 10089-10092.

DeLano, W.L. (2002). The PyMOL molecular graphics system. Delano Scientifc, San Carlos, CA, USA.

Donini, S., Ferrari, M., Fedeli, C., Faini, M., Lamberto, I., Marletta, A. S., Mellini, L., Panini, M., Percudani, R., Pollegioni, L., et al. (2009). Recombinant production of eight human cytosolic aminotransferases and assessment of their potential involvement in glyoxylate metabolism. Biochem J 422, 265-272.

Endo, F. (1998). Hereditary tyrosinemia type II. Ryoikibetsu Shokogun Shirizu, 134-136.

Essmann, U., Perera, L., Berkowitz, M.L., Darden, T., Lee, H., and Pedersen, L.G. (1995). A smooth particle mesh Ewald method. J Chem Phys 103, 8577-8593.

Federici, G., Di Cola, D., Sacchetta, P., Di llio, C., Del Boccio, G., and
Polidoro, G. (1978). Reversible inactivation of tyrosine aminotransferase from guinea pig liver by thiol and disulfide compounds. Biochem Biophys Res Commun 81, 650-655.

Fu, L., Dong, S.S., Xie, Y.W., Tai, L.S., Chen, L., Kong, K.L., Man, K., Xie, D., Li, Y., Cheng, Y., et al. (2010). Down-regulation of tyrosine aminotransferase at a frequently deleted region $16 q 22$ contributes to the pathogenesis of hepatocellular carcinoma. Hepatology 51 , 1624-1634.

Grishin, N.V., Phillips, M.A., and Goldsmith, E.J. (1995). Modeling of the spatial structure of eukaryotic ornithine decarboxylases. Protein Sci 4, 1291-1304.

Gross-Mesilaty, S., Hargrove, J.L., and Ciechanover, A. (1997). Degradation of tyrosine aminotransferase (TAT) via the ubiquitinproteasome pathway. FEBS Lett 405, 175-180.

Han, Q., Cai, T., Tagle, D.A., and Li, J. (2010). Structure, expression, and function of kynurenine aminotransferases in human and rodent brains. Cell Mol Life Sci 67, 353-368.

Han, Q., Fang, J., and Li, J. (2001). Kynurenine aminotransferase and glutamine transaminase $\mathrm{K}$ of Escherichia coli: identity with aspartate aminotransferase. Biochem J 360, 617-623.

Han, Q., Robinson, H., Cai, T., Tagle, D.A., and Li, J. (2009). Biochemical and structural properties of mouse kynurenine aminotransferase III. Mol Cell Biol 29, 784-793.

Hargrove, J.L., and Mackin, R.B. (1984). Organ specificity of glucocorticoid-sensitive tyrosine aminotransferase. Separation from aspartate aminotransferase isoenzymes. J Biol Chem 259, 386-393.

Hess, B., Bekker, H., Berendsen, H.J.C., and Fraaije, J.G.E.M. (1997). LINCS: a linear constraint solver for molecular simulations. J Comput Chem 18, 1463-1472.

Hess, B., Kutzner, C., van der Spoel, D., and Lindahl, E. (2008). GROMACS 4: Algorithms for Highly Efficient, Load-Balanced, and Scalable Molecular Simulation. J Chem Theory Comput 4, $435-447$.

Holm, L., and Sander, C. (1993). Protein structure comparison by alignment of distance matrices. J Mol Biol 233, 123-138.

Hoover, W.G. (1985). Canonical dynamics: Equilibrium phase-space distributions. Phys Rev A 31, 1695-1697.

Huey, R., Morris, G.M., Olson, A.J., and Goodsell, D.S. (2007). A semiempirical free energy force field with charge-based desolvation. J Comput Chem 28, 1145-1152.

Jansonius, J.N. (1998). Structure, evolution and action of vitamin B6dependent enzymes. Curr Opin Struct Biol 8, 759-769.

Käck, H., Sandmark, J., Gibson, K., Schneider, G., and Lindqvist, Y. (1999). Crystal structure of diaminopelargonic acid synthase: evolutionary relationships between pyridoxal-5'-phosphate-dependent enzymes. J Mol Biol 291, 857-876.

Ko, T.P., Wu, S.P., Yang, W.Z., Tsai, H., and Yuan, H.S. (1999). Crystallization and preliminary crystallographic analysis of the Escherichia coli tyrosine aminotransferase. Acta Crystallogr D Biol Crystallogr 55, 1474-1477.

Krissinel, E., and Henrick, K. (2004). Secondary-structure matching (SSM), a new tool for fast protein structure alignment in three dimensions. Acta Crystallogr D Biol Crystallogr 60, 2256-2268.

Laskowski, R.A., Macarthur, M.W., Moss, D.S., and Thornton, J.M. (1993). Procheck - a program to check the stereochemical quality of protein structures. J Appl Cryst 26, 283-291.

Macsai, M.S., Schwartz, T.L., Hinkle, D., Hummel, M.B., Mulhern, M. G., and Rootman, D. (2001). Tyrosinemia type II: nine cases of 
ocular signs and symptoms. Am J Ophthalmol 132, 522-527.

Maydan, G., Andresen, B.S., Madsen, P.P., Zeigler, M., RaasRothschild, A., Zlotogorski, A., Gutman, A., and Korman, S.H. (2006). TAT gene mutation analysis in three Palestinian kindreds with oculocutaneous tyrosinaemia type II; characterization of a silent exonic transversion that causes complete missplicing by exon 11 skipping. J Inherit Metab Dis 29, 620-626.

Mehta, P.K., Hale, T.I., and Christen, P. (1993). Aminotransferases: demonstration of homology and division into evolutionary subgroups. Eur J Biochem 214, 549-561.

Meissner, T., Betz, R.C., Pasternack, S.M., Eigelshoven, S., Ruzicka, T., Kruse, R., Laitenberger, G., and Mayatepek, E. (2008). RichnerHanhart syndrome detected by expanded newborn screening. Pediatr Dermatol 25, 378-380.

Minami-Hori, M., Ishida-Yamamoto, A., Katoh, N., Takahashi, H., and lizuka, H. (2006). Richner-Hanhart syndrome: report of a case with a novel mutation of tyrosine aminotransferase. J Dermatol Sci 41 , 82-84.

Morris, G.M., Goodsell, D.S., Halliday, R.S., Huey, R., Hart, W.E., Belew, R.K., and Olson, A.J. (1998). Automated docking using a Lamarckian genetic algorithm and an empirical binding free energy function. J Comput Chem 19, 1639-1662.

Murshudov, G.N., Vagin, A.A., and Dodson, E.J. (1997). Refinement of macromolecular structures by the maximum-likelihood method. Acta Crystallogr D Biol Crystallogr 53, 240-255.

Natt, E., Kida, K., Odievre, M., Di Rocco, M., and Scherer, G. (1992). Point mutations in the tyrosine aminotransferase gene in tyrosinemia type II. Proc Natl Acad Sci U S A 89, 9297-9301.

Natt, E., Westphal, E.M., Toth-Fejel, S.E., Magenis, R.E., Buist, N.R., Rettenmeier, R., and Scherer, G. (1987). Inherited and de novo deletion of the tyrosine aminotransferase gene locus at $16 q 22.1-q 22.3$ in a patient with tyrosinemia type II. Hum Genet 77, 352-358.

Nose, S. (1984). A unified formulation of the constant-temperature molecular-dynamics methods. J Chem Phys 81, 511-519.

Nose, S., and Klein, M.L. (1983). Constant pressure molecular dynamics for molecular systems. Mol Phys 50, 1055-1076.

Nowicki, C., Hunter, G.R., Montemartini-Kalisz, M., Blankenfeldt, W., Hecht, H., and Kalisz, H.M. (2001). Recombinant tyrosine aminotransferase from Trypanosoma cruzi: structural characterization and site directed mutagenesis of a broad substrate specificity enzyme. Biochim Biophys Acta 1546, 268-281.
Oostenbrink, C., Villa, A., Mark, A.E., and van Gunsteren, W.F. (2004). A biomolecular force field based on the free enthalpy of hydration and solvation: the GROMOS force-field parameter sets 53A5 and 53A6. J Comput Chem 25, 1656-1676.

Otwinowski, Z., and Minor, W. (1997). Processing of X-ray diffraction data collected in oscillation mode. Methods Enzymol 276, 307-326.

Parrinello, M., and Rahman, A. (1981). Polymorphic transitions in single crystals: A new molecular dynamics method. J Appl Phys 52, 7182-7190.

Pasternack, S.M., Betz, R.C., Brandrup, F., Gade, E.F., Clemmensen, O., Lund, A.M., Christensen, E., and Bygum, A. (2009). Identification of two new mutations in the TAT gene in a Danish family with tyrosinaemia type II. Br J Dermatol 160, 704-706.

Pettersen, E.F., Goddard, T.D., Huang, C.C., Couch, G.S., Greenblatt, D.M., Meng, E.C., and Ferrin, T.E. (2004). UCSF Chimera-a visualization system for exploratory research and analysis. J Comput Chem 25, 1605-1612.

Rego, J.V., Murta, S.M., Nirdé, P., Nogueira, F.B., de Andrade, H.M., and Romanha, A.J. (2008). Trypanosoma cruzi: characterisation of the gene encoding tyrosine aminotransferase in benznidazoleresistant and susceptible populations. Exp Parasitol 118, 111-117.

Rossi, F., Han, Q., Li, J., Li, J., and Rizzi, M. (2004). Crystal structure of human kynurenine aminotransferase I. J Biol Chem 279, 50214-50220.

Schneider, G., Käck, H., and Lindqvist, Y. (2000). The manifold of vitamin B6 dependent enzymes. Structure 8, R1-R6.

Sivaraman, S., and Kirsch, J.F. (2006). The narrow substrate specificity of human tyrosine aminotransferase-the enzyme deficient in tyrosinemia type II. FEBS J 273, 1920-1929.

Sobrado, V.R., Montemartini-Kalisz, M., Kalisz, H.M., De La Fuente, M.C., Hecht, H.J., and Nowicki, C. (2003). Involvement of conserved asparagine and arginine residues from the $\mathrm{N}$-terminal region in the catalytic mechanism of rat liver and Trypanosoma cruzi tyrosine aminotransferases. Protein Sci 12, 1039-1050.

Touboul, T., Hannan, N.R., Corbineau, S., Martinez, A., Martinet, C., Branchereau, S., Mainot, S., Strick-Marchand, H., Pedersen, R., Di Santo, J., et al. (2010). Generation of functional hepatocytes from human embryonic stem cells under chemically defined conditions that recapitulate liver development. Hepatology 51, 1754-1765.

Vagin, A., and Teplyakov, A. (1997). MOLREP: an automated program for molecular replacement. J Appl Cryst 30, 1022-1025. 